\title{
Phase retrieval from experimental far-field intensity data
}

\author{
M. J. Pérez-Ilzarbe, M. Nieto-Vesperinas, and R. Navarro \\ Instituto de Optica, Consejo Superior de Investigaciones Científicas, Serrano 121, 28006 Madrid, Spain
}

Received July 7, 1989; accepted October 23, 1989

\begin{abstract}
We demonstrate the reconstruction of real and positive objects from experimental far-field intensity measurements by means of two phase-retrieval algorithms. Both the iterative Fourier transform and the simulated annealing algorithms are used, and an analysis is made of the advantages and disadvantages of each of these procedures and also of combinations of both methods. The objects tested either were binary or had many gray levels. We worked with data with a considerable amount of experimental noise, and in addition we recognized the importance of taking into account the nonrandom distortions produced by the detecting devices, which can critically bias the results toward erroneous estimates of the objects. Noisy data, however, can create ambiguities in the reconstructions, and hence additional information may be needed to overcome this disadvantage.
\end{abstract}

\section{INTRODUCTION}

Phase retrieval is an important problem in many areas of physics, e.g. optics, x-ray diffraction, scattering, and astronomy. ${ }^{1-11}$

Recently a simulated annealing (SA) algorithm was proposed $^{12}$ for phase retrieval from the power spectrum of a real and positive object of finite support, as in the case of astronomical images ${ }^{13-18}$ and in diffractive optics for real objects. ${ }^{19,20}$ This method has been further improved and successfully used in computer simulations of object reconstruction with different signal-to-noise ratios ${ }^{21}$ and also of photon-limited stellar speckle interferometry. ${ }^{18}$ The algorithm is flexible and permits an easy introduction of additional constraints. Its performance is uniformly good. However, it requires a rather lengthy computing time. In some cases, it can be accelerated by first using the hybrid input-output version of the iterative Fourier-transform (IFT) algorithm. ${ }^{18,21-23}$

In this paper we demonstrate the reconstruction of real and positive objects from direct experimental measurements of their power spectra by using the SA technique. The only $a$ priori constraints used are that the object be real and positive. The object is reconstructed inside a square window having half the side of its autocorrelation. No additional constraints, such as the shape of the support or symmetry of the object, are imposed. Comparisons are also made with the IFT algorithm, which we have also used directly in some tests, and with combinations of both the SA and IFT methods. These combinations are used either to improve the results of the IFT algorithm or to save computing time for the SA algorithm in some cases.

Reconstructions of a diffuse complex object of known support from experimental far-field measurements by using the IFT algorithm have been reported. ${ }^{24}$ Also, reconstructions of real and positive objects with this algorithm have been done both from laboratory simulations of stellar speckle interferometry ${ }^{25,26}$ and from measurements of backscattered laser speckle intensity patterns ${ }^{27}$ made by using the imaging correlography technique. ${ }^{28,29}$ While those references deal with processing of specklegrams that yield the power spectrum of a real and positive image, we propose a rather differ- ent and crude experiment, namely, the direct measurement of the far-field diffraction pattern (power spectrum) of a real and positive image. This experiment entails great difficulty in the estimation of the power spectrum owing to the large central peak in the diffraction pattern that is often present. The consequence is an important amount of noise in the data, which challenges the efficiency of phase-retrieval algorithms. The experiment that we present shows the usefulness of these methods in situations in which only information about the power spectrum of a real and positive object is obtained. Of course, in experiments such as those performed in Refs. 25-29, the algorithm proposed here can be envisaged as an alternative procedure.

\section{EXPERIMENTAL PROCEDURE}

The determination of the object's power spectrum implies knowledge of the object's autocorrelation. Two different optical and digital processes have been used to get the autocorrelation data. They depend on the kinds of test objects to be used, that is, either we deal with binary objects whose transmittance is either zero or one or we work with objects with a transmittance having a large number of values, i.e., characterized by a large number of gray levels.

\section{Binary Objects}

The experimental setup is shown in Fig. 1. It is as follows: a spatially filtered and collimated $\mathrm{He}-\mathrm{Ne}$ laser beam $(\lambda=$ $0.633 \mu \mathrm{m}$ ) illuminates the object, which is a binary mask constructed by punching holes through a metallic plate. The object is placed in the pupil plane of a lens of 1-m focal length, which forms the Fourier transform of the object in the back focal plane. The power spectrum is detected by a TV camera, which is connected to a 8-bit frame grabber. Digitization is made on a selected window of $128 \times 128$ pixels. The measured size of each pixel given by the camera-plus-digitizer system is $17 \mu \mathrm{m} \times 17 \mu \mathrm{m}$ (with an estimated error smaller than $5 \%$ ). However, the effective resolution of the system is poor, so oversampling the images of the power spectra is recommended. From the dimensions of the window and the sampling spacing in the Fourier plane, and taking into account both the focal length of the lens and the 
laser wavelength, we can calculate the size $s$ of the pixel in the object plane, i.e., $s \approx 0.3 \mathrm{~mm} \times 0.3 \mathrm{~mm}$. The object is included in a $9.6 \mathrm{~mm} \times 9.6 \mathrm{~mm}$ square, i.e., its support is inside a window of $32 \times 32$ pixels. The power spectrum is sampled at twice the minimum rate (Nyquist rate) to avoid aliasing.

The simple, low-cost RCA Model TC2000 closed-circuit TV camera degrades the image during the detection process, mainly in four different ways: random noise, additive bias, nonlinearity, and nonconstant frequency response. The importance of these effects is estimated, and a restoration process is followed to attempt to minimize their influence.

The additive bias (nonzero intensity detected in a dark frame) has a mean value of 30 in the $0-225$ range of gray levels of the 8-bit digital-to-analog converter of the frame grabber. This bias is not spatially uniform over the entire window but varies between 28 and 32 . Then the actual intensity range that can be detected decreases from the 8-bit theoretical range from 0 to 255 to the range from 0 to $\sim 225$. The random noise is estimated by calculating (over 100 frames) the standard deviation of the measured intensity at each pixel $\sigma\left(I_{i j}\right)$ as a function of its mean value, $I_{i j}$. We have found that $\sigma$ does not actually depend on the intensity value $I_{i j}$ of the particular pixel $(i, j)$ and is thus a constant, $\sigma \approx 2.9$. Then, defining the signal-to-noise ratio (SNR) as usual,

$$
\operatorname{SNR}\left(I_{i j}\right)=I / \sigma\left(I_{i j}\right)=I / \sigma
$$

we have a linear dependence of SNR on $I_{i j}$, in this case $\operatorname{SNR}\left(I_{i j}\right) \approx 0.34 \times I_{i j}$.

To reduce the noise we average ten frames of each power spectrum recorded. We estimate the SNR, as before, over 100 frames, but now each of these frames is, in turn, the average of ten frames. We have found that $\sigma \approx 0.9$ and $\operatorname{SNR}\left(I_{i j}\right) \approx 1.1 \times I_{\mathrm{ij}}$. Then we substract the average of ten dark frames to correct the bias; negative values of this averaged power spectrum are set to zero, and positive ones below a certain threshold are also set to zero. In this way we keep the total energy constant.

An intensity calibration of the camera is made by taking a curve (in this case a parabola) that is used for correction of the nonlinearity. This type of nonlinear response of the camera tends to raise the low-intensity areas of the image and to lower the high-intensity ones, thus magnifying the background of the detected power spectrum. Although this is not an especially disturbing effect, better results are obtained if we correct it.

The frequency response is controlled by measuring, in two orthogonal directions, the power spectrum of a row of holes of $0.5-\mathrm{mm}$ diameter with $0.5-\mathrm{mm}$ separation. The autocorrelation function of this test was evaluated. The modulation transfer function (MTF) of the camera appeared to multiply this function. This MTF was estimated by assuming circular symmetry and corresponds to an effective resolution of $\sim 24 \mu \mathrm{m}$. Once the MTF was found, the correction was made by applying the corresponding inverse filter. This was done after cleaning the object autocorrelation by establishing a threshold to set to zero those values of the autocorrelation that are low and comparable with the noise. We have found that this correction was necessary even though the effect of frequency response (resolution) of the camera had been attenuated by oversampling the power spectra.

The resulting autocorrelations are contained in a window of $63 \times 63$ pixels. Thus the object is bounded by a window of $32 \times 32$ pixels. No other $a$ priori knowledge about the object support is assumed. In addition, nonnegativity is also presupposed.

Objects Characterized by a Large Number of Gray Levels The object consists of a picture recorded in a photographic film and is immersed in a liquid gate in order to compensate for the phase effects due to variations in emulsion thickness.

With these types of object, the 256 intensity levels of the frame grabber are not enough to describe the power spectrum, which in this case generally has a bright central peak much more intense than the rest of the Fourier transform. This is the main problem in detecting the power spectrum of an object with many gray levels. It is necessary to use a filter that eliminates the peak, in order to be able to detect some intermediate frequency information. The high-frequency information is unavoidably lost.

The experimental setup is shown in Fig. 2. The part of the apparatus shown at the left is the same as that used with

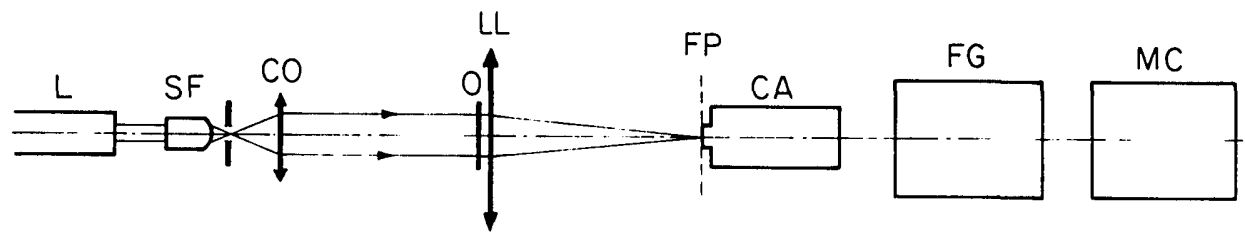

Fig. 1. Experimental setup for binary objects: L, laser; SF, spatial filter; CO, collimator; O, object; LL, Fourier-transforming lens; FP, Fourier plane; CA, camera; FG, frame grabber; MC, microcomputer.



Fig. 2. Experimental setup for objects with several gray levels: L, laser; NF, neutral filter; SF, spatial filter; CO, collimator; O, object; LL, Fourier-transforming lens; FP, Fourier plane; $L_{1}$, lens; $P_{1}$, image plane of $L_{1} ; F$, filter on axis; $L_{2}$, lens; $P_{2}$, image plane of $L_{2} ; C A$, camera; $F G$, frame grabber; $\mathrm{MC}$, microcomputer. 
binary objects, but in this case lens $\mathrm{L}_{1}$, of $75-\mathrm{mm}$ focal length, forms an intermediate image of the spectrum in plane $P_{1}$, where a filter is placed on the optical axis. This filter is a black (i.e., has a high neutral optical density) stop of $250-\mu \mathrm{m}$ diameter, constructed photographically and also immersed in a liquid gate. This intermediate image allows the size of the central spot of the spectrum to be fitted to that of the filter. Then lens $\mathrm{L}_{2}$, of $75-\mathrm{mm}$ focal length, makes a second image of the object Fourier transform without the central peak in plane $P_{2}$, where the camera is placed for detection. The magnification of this image is chosen such that it provides the desired size of the spectrum in the detection plane. We grab this image in a first frame.

Then we make another image of the same spectrum, this time removing the black stop filter. In this frame only a bright peak is visible. This is done by placing appropriate neutral-density filters in the laser beam to attenuate the central peak of the spectrum, so that now this peak lies inside the detectable dynamic range of the camera. By grabbing this image in a second frame, and by knowing the absorption rate of the neutral-density filter used, it is possible to reconstruct the whole power spectrum from the two frames containing the central peak and the intermediatefrequency intensity values, respectively.

A Sony Model AVC-D5CE charge-coupled device (CCD) camera is used in this case. This camera produces no additive bias. However, a dark frame is also subtracted now from each power spectrum recorded, in order to prevent the detection of parasitic light. Random noise is estimated in the same way as before: $\sigma$ varying from $\sim 1.4$ at the highest intensity levels to $\sim 2.6$ at low levels. The SNR then varies from $\sim 0.7 \times I_{i j}$ to $\sim 0.4 \times I_{i j}$. Because this SNR value is so high, we considered it unnecessary to average frames.

The intensity response of the camera was tested, and, although it is not perfectly linear, the CCD does not produce so clear a bias as the vidicon camera does, thus raising the background of the image. Thus we believe that it is not necessary to correct the power-spectrum data for nonlinear effects, and we show this in the results described below.

An estimation of the camera frequency response showed us that it is not constant, as in the case of the TV camera. However, a different procedure is now followed to minimize this effect.

Oversampling of the power spectrum at several times the Nyquist rate involves a larger window in the object plane and therefore results in expanding the camera MTF over a broader frame. Since, however, the size of the object autocorrelation remains the same, and since it is located in the central area of the window, this object autocorrelation function is multiplied by the central part of the camera MTF, which, within this area, can be considered approximately constant.

Nevertheless, handling such large windows could produce time and memory computing problems. Nonetheless, we can choose the magnification of the second image of the spectrum such that it is oversampled by at least six times the Nyquist rate, within a window of $512 \times 512$ pixels, and then subsample it during the detection process with the aid of the microcomputer, keeping overall a resultant oversampling of twice the Nyquist rate, as in the case of binary objects.

This procedure has another advantage; the CCD camera gives samples that are not equally spaced in the vertical and horizontal directions. The sampling spacing is 14.5 and 10.5 $\mu \mathrm{m}$ (error smaller than $5 \%$ ) in the horizontal and vertical directions, respectively. To obtain a square sampling we subsample the broad $512 \times 512$ power spectrum, keeping a value for every three pixels in the horizontal direction and every four pixels in the vertical direction. In this way we obtain a frame with the power spectrum in a final window of $128 \times 128$ pixels, where the sampling is almost equally spaced in both directions $(\sim 43.5 \mu \mathrm{m}$ in the horizontal direction and $42 \mu \mathrm{m}$ in the vertical direction), and the effect of camera frequency response is negligible.

To calculate the pixel size $s$ in the object space, we must now take into account the total magnification applied to the original spectrum to obtain its second image (the resulting size is approximate, since we do not know this magnification exactly), the window dimensions and the pixel size in the Fourier space (we put $s \approx 42.5 \mu \mathrm{m} \times 42.5 \mu \mathrm{m}$ for this calculation), and also the laser wavelength and the focal length of the Fourier-transforming lens. Our calculations indicate that $s \approx 0.2 \mathrm{~mm} \times 0.2 \mathrm{~mm}$, i.e., the object must be contained in a square of $6.4 \mathrm{~mm} \times 6.4 \mathrm{~mm}$ in order to have its support inside a window of $32 \times 32$ pixels. The power spectrum is then sampled at twice the Nyquist rate, as desired.

Fourier transforming this power spectrum, we obtain the object autocorrelation function, which appears to be surrounded by low negative and positive values all over the window. We clean it by setting to zero all values from the area where the function is everywhere positive. The final autocorrelation results in a square of $63 \times 63$ pixels, and no other $a$ priori knowledge about its support is assumed.

\section{NUMERICAL PROCEDURE AND RESULTS}

Figures 3(a) and 4(a) show two different binary test objects. The corresponding measured power spectrum, averaged over ten frames but without the other corrections described above having been made, are shown in Figs. 3(b) and 4(b). Note that the power spectrum of Fig. 3(b) is more thoroughly described with the intensity range available to the camera than is the power spectrum of Fig. 4(b). On the other hand, Figs. 3(c) and 4(c) show the autocorrelation functions constructed from the power spectra of Figs. 3(b) and 4(b), respectively.

Both the IFT and the SA algorithms were run in a CCD Cyber $/ 855$ computer to reconstruct the objects of Figs. 3(a) and 4(a) from the power spectra of Figs. 3(b) and 4(b), respectively. This was been done after the corrections described in Section 3 were applied to the experimental recordings. The autocorrelation data used for SA, as well as the power-spectrum data used for the IFT, are normalized to unity. The starting guess is a random object constructed from the halved autocorrelation, according to the description reported in Ref. 9; it is scaled so that its autocorrelation is also normalized to unity. This saves time for the performance of the SA algorithm, since this guess has an $F$ error [see Ref. 15] that is not high, allowing us to start at a relatively low temperature and then to avoid unnecessary cycles. The $F$ error is the quantity that is minimized by the SA algorithm, and in the $k$ th iteration it is defined as follows ${ }^{12}$ :

$$
F_{k}=\frac{1}{C}\left[\sum_{i=1}^{2 N-1} \sum_{j \leq i}^{2 N-1} r_{i j}^{(k) 2}\right]^{1 / 2},
$$




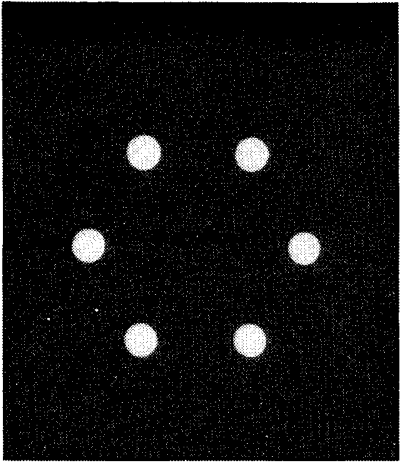

(a)

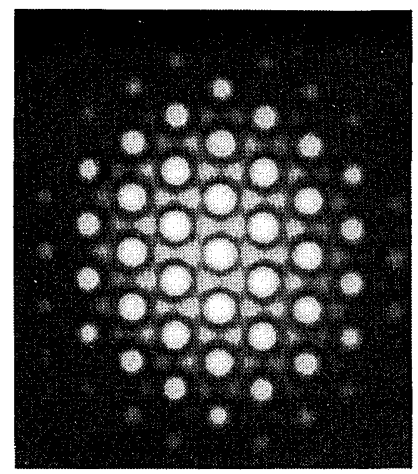

(b)

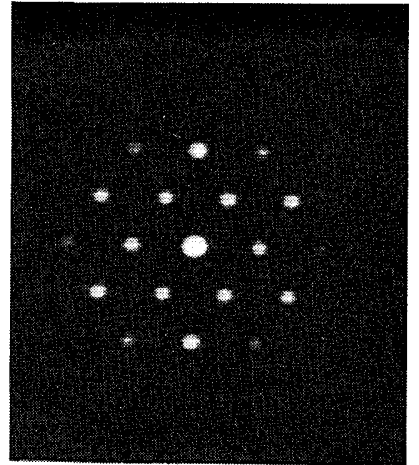

(c)

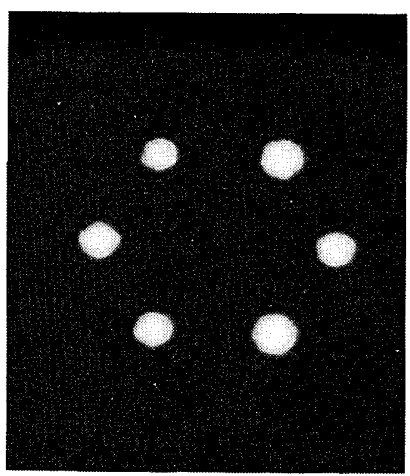

(d)
Fig. 3. (a) Binary object, (b) measured power spectrum, (c) autocorrelation, (d) quenched IFT reconstruction.

where $r_{i j}{ }^{(k)}$ are the residuals:

$$
\begin{gathered}
r_{i j}{ }^{(k)}=Q_{i j}-\sum_{m=1}^{N} \sum_{n=1}^{N} f_{m n}{ }^{(k)} f_{m+i-N, n+j-N}{ }^{(k)}, \\
1 \leq i \leq 2 N-1, \quad 1 \leq j \leq 2 N-1,
\end{gathered}
$$

and $C$ is the normalization constant:

$$
C=\left(N^{2}+1\right) / 2 .
$$

$Q_{i j}$ is the value of the normalized autocorrelation data at the pixel $(i, j), f_{m n}{ }^{(k)}$ is the value of the object estimate at the pixel $(m, n)$ in the $k$ th iteration, and $N$ is the linear dimension of the object (then $C$ is the number of pixels of the autocorrelation).

The IFT schedule consists of 7 cycles, each having 20 error-reduction plus 100 input-output iterations, ending with 20 iterations of the error reduction (see Ref. 9). The quantity that is minimized by this algorithm is the $E$ error, which in the $k$ th iteration is defined as follows ${ }^{9}$ :

$$
E_{k}=\frac{\sum_{m, n \in \Gamma}\left|g_{m n}^{(k)}\right|^{2}}{\sum_{m, n}\left|g^{\prime(k)}{ }_{m n}\right|^{2}},
$$

where $g_{m n}^{\prime}{ }^{(k)}$ is the value of the object estimate at the pixel $(m, n)$ after the $k$ th iteration and $\Gamma$ is the set of points at which $g_{m n}^{\prime}{ }^{(k)}$ violates the constraints, which in our case are the upper bound of support $(32 \times 32$ pixels $)$ and positivity.
For the object of Fig. 3(a) the IFT arrived at a satisfactory result in $8 \mathrm{~min}$ of CPU time. The $E$ error decreased from $E_{0}$ $=0.625$ for the initial iteration to $E_{f}=0.136$ for the last iteration. The corresponding $F$ error was $F_{f}=1.71 \times 10^{-4}$. No further decrease was attained by adding more iterations. The SA yielded a similar reconstruction but required $\sim 40$ min of CPU time. A quenched version (i.e., at temperature $T=0$ ) of the SA (see Ref. 15) applied to the IFT result decreased the $F$ error to $F_{f}^{\prime}=7.05 \times 10^{-5}$ in $3 \mathrm{~min}$ of CPU time. The resulting image is sharper and is shown in Fig. $3(d)$.

The object of Fig. 4(a) was reconstructed with the SA method. The $F$ error of the initial guess was $F_{0}=1.53 \times$ $10^{-3}$; that for the final iteration was $F_{f}=1.97 \times 10^{-4}$. The temperature decay schedule used was $T_{n+1}=0.75 \times T_{n}$. The initial temperature was chosen to be $T_{0}=0.075$, and the $F$ error stagnated at $T_{f}=5.7 \times 10^{-7}$. The bound $\alpha$ (see Ref. 24) for the perturbation of each pixel intensity was kept proportional to the current $F$ error by means of the relation $\alpha$ $=12 \times F$. A uniformly distributed random-number generator is used for pixel perturbation. The reconstruction is shown in Fig. 4(f). The process required $90 \mathrm{~min}$ of $\mathrm{CPU}$ time, but in $\sim 50$ min an acceptable but less clear reconstruction can also be obtained. We were unable to obtain a performance of the IFT similar to this result for this object. The initial $E$ error was $E_{0}=0.52$, and the final one was $E_{f}=$ 0.153 , corresponding to $F_{f}=4.51 \times 10^{-4}$, stagnating in an estimate of the object with a worse visual appearance than



(a)

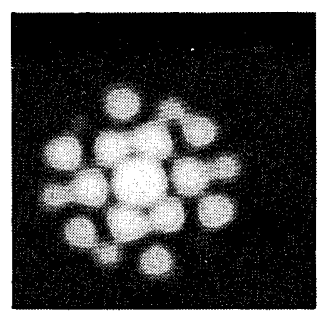

(b)

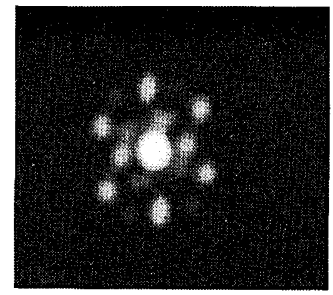

(c)

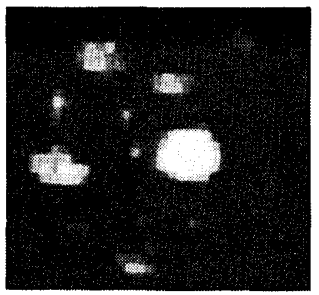

(d)

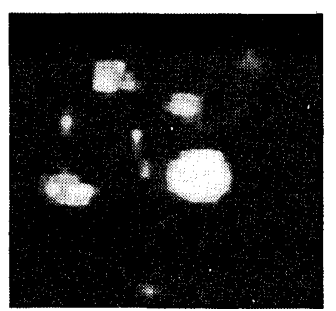

(e)

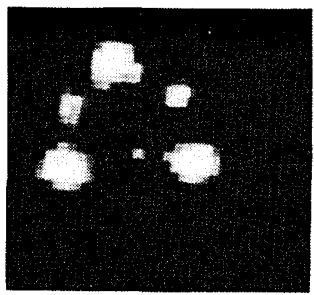

(f)
Fig. 4. (a) Binary object, (b) measured power spectrum, (c) autocorrelation, (d) IFT result, (e) quenched IFT result, (f) SA reconstruction. 
that obtained with the SA reconstruction, as can be seen from Fig. 4(d).

However, by quenching this estimate during $3 \mathrm{~min}$ of CPU time, we obtain a cleaner image, which can be seen in Fig. 4(e). This result has the general features of that for the IFT but has a cost function of $F_{f}=1.61 \times 10^{-4}$. This cost function is of the same order of, and even lower than, that of the SA result (remember that this value was $1.97 \times 10^{-4}$ ). The results are two different possible solutions compatible with the same amount of noise in the data. Even with the SA algorithm, and using other schedules or other recorded power spectra as initial data, we have obtained other results with cost functions comparable with that of the result of Fig. 4(f) but having different structure. Therefore the criterion that we have chosen with which to assess the SA result as the best one in this case is merely subjective, because we know the visual appearance of the true object. Had we ignored this true object, as one must always do in real cases, some additional $a$ priori information or some criterion of choice would be needed to eliminate this or other possible ambiguities. This example confirms, therefore, the possible occurrence of nonuniqueness in phase retrieval in two dimensions from noisy power-spectrum data in real cases (as pointed out previously ${ }^{12,14,23}$ ). This corresponds to the possibility of approximate factorization of the autocorrelation polynomi$\mathrm{al}^{3,4,8,10}$ when there is some uncertainty interval in its coefficients. ${ }^{30}$

These two examples show, therefore, that while the IFT may be preferable from the point of view of speed, the SA, either alone or combined with the IFT, is a useful alternative when the IFT stagnates.

A test object with many gray levels is shown in Fig. 5(a). Here the square root of the intensity distribution, as detected by the camera, is taken to show the amplitude distribution of the object. Its corresponding power spectrum, constructed from the two frames containing the low- and intermediate-frequency measured data as described in Section 3, is shown in Fig. 5(c). It is shown in logarithmic scale to compress the intensity range to 255 levels, and its size is magnified by a factor of 1.2 with respect to the other power spectra shown in order to make its structure visible. There is an appreciable loss of high-frequency information in these data. The autocorrelation function constructed from this power spectrum is shown in Fig. 5(d).

The result reconstructed from these data with the IFT method is shown in Fig. 5(e). The initial $E$ error is $E_{0}=0.27$, and the final $E$ and $F$ errors are $E_{f}=0.086$ and $F_{f}=2.37 \times$ $10^{-4}$, respectively. The general features of the object are clearly visible in this result; the intensities of the maxima are quite inaccurate, however. Quenching the IFT solution did not improve the reconstruction in this case. On the contrary, the result obtained has a visual appearance that is worse than that of the input, even though the $F$ error drops. This is because the cost function, which depends on the noisy input data, is a measure of the agreement of the solution with these data, but it is not a criterion of the agreement of these data with the ideal noise-free data corresponding to the true object.

A single run of the SA, starting with a random guess with an $F$ error of $8.11 \times 10^{-4}$ at $T_{0}=0.008$ and ending at $T_{f}=3.5$ $\times 10^{-7}$, gives a reconstruction with an $F$ error of $2.73 \times 10^{-5}$ and takes $\sim 75$ min of CPU time; the result can be seen in Fig.

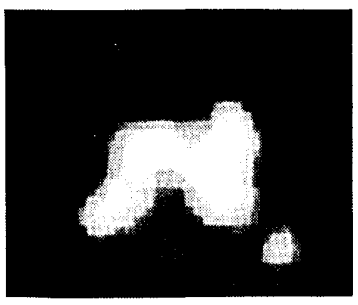

(a)

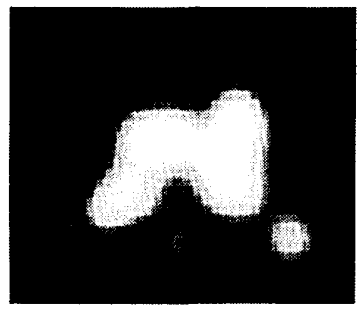

(b)

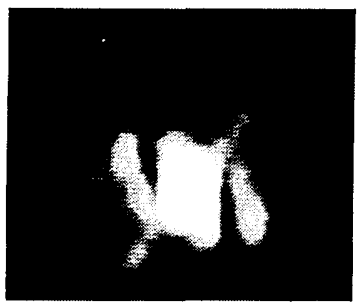

(c)

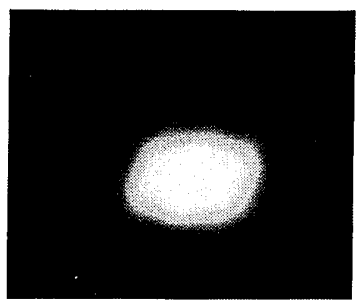

(d)

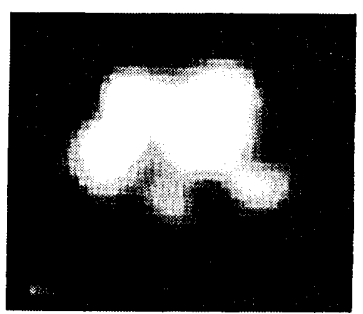

(e)

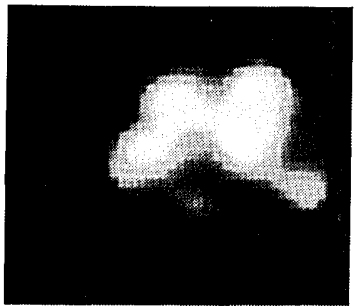

(f)
Fig. 5. (a) Object with several gray levels, (b) filtered object, (c) power spectrum constructed from the two measured frames containing the low- and intermediate-frequency data (shown in logarithmic scale), (d) autocorrelation, (e) IFT reconstruction, (f) SA reconstruction.

5(f). The pixel perturbation bound (see Ref. 24) used was $\alpha$ $=18 \times F$. It shows a loss of resolution due to the loss of highfrequency information on the power-spectrum data. We have simulated this loss of resolution by applying to the object [Fig. 5(a)] a filter that sets to zero all values of the object's Fourier transform corresponding to the values of the power spectrum that are below the minimum intensity level detected by the camera. The object filtered is shown in Fig. 5 (b) for comparison with the reconstructions.

To obtain a result similar to that of the SA algorithm by introducing the IFT solution as input guess, it is necessary to start at quite a high temperature. But then the computing time is not much shorter than that needed for a single run of the SA. Although for this object there is not so a severe problem of ambiguity as in the case of the object of Fig. 4(a), some differences exist in the several solutions obtained with different combinations of the two algorithms, all of them compatible with the input data.

Fast SA (FSA) versions ${ }^{31}$ of the reconstruction method were also attempted successfully, but no net advantage, either in performance or in computing time, was observed. These versions use a Cauchy random-number generator and permit the acceleration of the temperature decay without trapping of the $F$ error into local minima. However, in our case the computing time used by the algorithm depends both 
on the temperature schedule and on the number of cycles per constant temperature. We ran the FSA with the schedule $T_{n+1}=0.5 \times T_{n}$, obtaining results similar to those when the conventional SA was used; however, this does not save computing time since it takes longer to reach equilibrium at each temperature step.

Signal recovery of the object of Fig. 4(a), although the object has a binary intensity distribution, seems to be more difficult than for any of the other three objects presented in this paper. This could be because most of the corresponding measured power spectrum has a poorer SNR than the power spectra of Figs. 3(b) and 5(c).

In fact, to compare the SNR of these three power spectra we normalize to unity the intensity of each of them, obtaining the following expressions for the SNR of the $128 \times 128$ power spectra shown in Figs. 3(b), 4(b), and 5(c):

Power spectrum 3: $\operatorname{SNR}^{3}\left(I_{i j}{ }^{3}\right) \approx 241.1 \times I_{i j}{ }^{3} \quad$ [Fig. 3(b)], Power spectrum 4: $\operatorname{SNR}^{4}\left(I_{i j}{ }^{4}\right) \approx 241.8 \times I_{i j}{ }^{4} \quad$ [Fig. 4(b)], Power spectrum 5: $\operatorname{SNR}^{5}\left(I_{i j}{ }^{5}\right) \approx 915.7 \times I_{i j}{ }^{5} \quad$ [Fig. 5(c)], $1 \leq i \leq 128, \quad 1 \leq j \leq 128$, where $\mathrm{SNR}^{k}$ is the $\mathrm{SNR}$ in the power spectrum $k$ and $I_{i j}{ }^{k}$ is the corresponding normalized intensity detected at pixel $(i$, $j)$ of this power spectrum. In this calculation we have neglected the nonlinear response of the camera, and for power spectrum 5 we have considered the SNR in the zone corresponding to intermediate frequencies, assuming $\sigma$ constant and equal to 0.55 . As we can see, power spectrum 4 has the lowest SNR. Furthermore, the range of intermediate frequencies of a spectrum carries most of the information about the structure of the object. Therefore, although $\mathrm{SNR}^{3}$ and $\mathrm{SNR}^{4}$ are quite similar, we can note from Figs. 3(b) and 4(b) that, at the most meaningful frequencies, the intensity detected is lower in the power spectrum of Fig 4(b) than in the power spectrum of Fig. 3(b), i.e., the SNR is considerably lower.

There are other sources of error in the data, such as coherent noise in the optical system, the remaining nonrandom effects of the cameras, and errors in calibration. For the object in Fig. 5 it is possible that there are small phases in the object that are due to imperfections in the liquid gate; in addition, errors in constructing the power spectrum of Fig. 5(c) from the two frames detected as indicated above may be present (indeed, this operation must be done carefully). The coherent noise is greater in this power spectrum owing to the higher number of optical elements used in this case (see the experimental setup of Fig. 2). However, the procedure followed to get power spectrum 5 from two measured frames permits the detection of high-intensity signals in the range of intermediate frequencies. It is because of this fact that this spectrum has the highest SNR. Moreover, the highquality performance of the CCD camera, which eliminates the necessity for correcting the measured data, also improves the final accuracy of the autocorrelation and powerspectrum data used for phase retrieval. The results obtained seem to prove that the procedures followed are good enough to eliminate severe bias in the reconstructions and also that the two phase-retrieval methods used are robust enough to work successfully with noisy experimental data. Noisy data, however, can give rise to ambiguities in the reconstructions compatible with these data, and thus some additional $a$ priori information may be necessary.

\section{CONCLUSIONS}

We have studied the performance of both the SA and the IFT methods of phase retrieval from experimental far-field intensity data, corresponding to positive and real objects of finite support. The hybrid input-output version of the IFT algorithm gives an acceptable although sometimes not highly accurate estimation of the object. If the method stagnates, then it may be used as a starting guess for the SA method, which can then refine the result. This saves computing time; the SA algorithm alone can be run without the help of the IFT and arrive at a similar reconstruction, but this is more time consuming, although it permits more flexibility.

For binary objects, a quenched version of the SA algorithm applied to the IFT output can yield quite a good result in a few minutes of CPU time. When the IFT estimate is near a local minimum of the cost function, we have always observed that the SA algorithm used with a random starting guess is able to arrive at a good reconstruction in less than 90 min of CPU time. For the object with several gray levels with which we have worked, an acceptable estimate is found with the IFT. A better result is obtained with the SA algorithm starting with a random guess; however this involves 75 min of CPU time. The last solution is more accurate than the annealed IFT result when one compares the result with the object. However, in practical situations there is no objective criterion for choosing the SA solution as the best estimate of the object, since the agreement with the measured data is good and is similar in both of them. This is also true for the SA and the quenched IFT results obtained as estimators of the second binary object tested. For this particular object, the structural difference between the two results is great; therefore each reconstruction corresponds to a different solution of the phase-retrieval problem, and these solutions are equally compatible with the measured data. In all cases, the low intensity levels are not accurately reconstructed with either the IFT or the SA algorithm; these errors are unavoidable since they are determined by the errors in the autocorrelation or, equivalently, in the power-spectrum data.

A more sophisticated digital processing of the power-spectrum data before using the phase-retrieval algorithms (e.g., the Wiener filter used in Refs. 27-29) could certainly improve the final result, whose agreement with the corresponding object would be limited by the SNR in the measurements. We have also noted the importance of taking into account the nonrandom effects of the detection device in the intensity data. We realize that in the measurements done, and for the application in which we are interested, the most critical effect is the camera frequency response. Oversampling the power spectrum at several times the Nyquist rate reduces this effect. When it is not possible to do this, an estimation of the camera MTF and a consequent correction of the data may be needed. The results achieved, however, are satisfactory if one bears in mind the rather poor SNR as well as other important sources of error in the detected power spectra; thus the efficiency of the two algorithms is shown.

As we have seen, there is no general rule that can be given 
for reconstruction processes. From our observations we conclude that there is no automatic procedure, either. The best recommendation is to use both the IFT and the SA algorithms as described above, analyzing the decay of both the $E$ and the $F$ errors, until a consistent result is found from the different attempts. Nonetheless we believe that, in some cases, when different trials produce clearly different reconstructions with similar errors, some additional $a$ priori information will be needed, apart from the power-spectrum data (the solutions can be so different from each other that averaging them would be meaningless). This necessity has nothing to do with the convergence and performance of the algorithms but rather is related to the possible inherent nonuniqueness of the solution compatible with the noisy autocorrelation (or power spectrum). A greater guarantee of uniqueness could be obtained if there were only a small error in the autocorrelation data.

\section{ACKNOWLEDGMENTS}

This research was supported by the Comision Interministerial de Ciencia y Tecnología of Spain under grant pb0278. M. J. Pérez-Ilzarbe acknowledges a grant from Ministerio de Educación y Ciencia.

\section{REFERENCES}

1. A. Walther, "The question of phase retrieval in optics," Opt. Acta 10, 41-49 (1963).

2. R. H. T. Bates, "Fourier phase problems are uniquely solvable in more than one dimension. I: Underlying theory," Optik 61, 247-262 (1982); K. L. Garden and R. H. T. Bates, "Fourier phase problems are uniquely solvable in more than one dimension. II: One-dimensional considerations," Optik 62, 131-142 (1982); W. R. Fright and R. H. T. Bates, "Fourier phase problems are uniquely solvable in more than one dimension. III: Computational examples for two dimensions," Optik 62, 219230 (1982).

3. Yu. M. Bruck and L. G. Sodin, "On the ambiguity of the image reconstruction problem," Opt. Commun. 30, 304-308 (1979).

4. M. A. Fiddy, B. J. Brames, and J. C. Dainty, "Enforcing irreducibility for phase retrieval in two dimensions," Opt. Lett. 8, 96-98 (1983).

5. A. Levi and H. Stark, "Image restoration by the method of generalized projections with application to restoration from magnitude," J. Opt. Soc. Am. A 1, 932-943 (1984).

6. P. L. Van Hove, M. H. Hayes, J. S. Lim, and A. V. Oppenheim, "Signal reconstruction from Fourier transform magnitude," IEEE Trans. Acoust. Speech Signal Process. ASSP-31, 12861293 (1983).

7. K. Chalasinska-Macukov and H. H. Arsenault, "Fast iterative solution to exact equations for the two-dimensional phase-retrieval problem," J. Opt. Soc. Am. A 2, 46-50 (1985).

8. M. Nieto-Vesperinas and J. C. Dainty, "Testing for uniqueness of phase recovery in two dimensions," Opt. Commun. 52, 94-98 (1984).

9. J. R. Fienup, "Phase retrieval algorithms: a comparison," Appl. Opt. 21, 2758-2769 (1982).
10. J. L. C. Sanz, T. S. Huang, and F. Cukierman, "Stability of unique Fourier-transform phase reconstruction," J. Opt. Soc. Am. 73, 1442-1445 (1983).

11. M. Nieto-Vesperinas, "Dispersion relations in two dimensions: applications to the phase problem," Optik 56, 377-384 (1980).

12. M. Nieto-Vesperinas and J. A. Méndez, "Phase retrieval by Monte Carlo methods," Opt. Commun. 59, 249-254 (1986).

13. J. C. Dainty, "Stellar speckle interferometry," in Laser Speckle and Related Phenomena, 2nd ed., J. C. Dainty, ed. (SpringerVerlag, Berlin, 1984).

14. J. C. Dainty and J. R. Fienup, "Phase retrieval and image reconstruction for astronomy," in Image Recovery: Theory and Application, H. Stark, ed. (Academic, New York 1986).

15. G. R. Ayers, M. J. Northcott and J. C. Dainty, "Knox-Thompson and triple-correlation imaging through atmospheric turbulence," J. Opt. Soc. Am. A 5, 963-985 (1988).

16. J. R. Fienup, "Space object imaging through the turbulent atmosphere," Opt. Eng. 18, 529-534 (1979).

17. G.J. M. Aitken and R. Johnson, "Phase-gradient reconstruction from photon-limited stellar speckle images," Appl. Opt. 26, 4246-4249 (1987).

18. R. Navarro, F. J. Fuentes, and M. Nieto-Vesperinas, "Simulated annealing image reconstructed in photon-limited stellar speckle interferometry," Astron. Astrophys. 208, 374-380 (1989).

19. E. Wolf, "Three-dimensional structure determination of semitransparent objects from holographic data," Opt. Commun. 1, 153-156 (1969).

20. G. Ross, M. A. Fiddy, and M. Nieto-Vesperinas, "The inverse scattering problem in structural determinations," in Inverse Scattering Problems in Optics, H. P. Baltes, ed., Vol. 20 of Topics in Current Physics (Springer-Verlag, Berlin, 1980), pp. $15-71$.

21. M. Nieto-Vesperinas, R. Navarro, and F. J. Fuentes, "Performance of a simulated annealing algorithm for phase retrieval," $\mathrm{J}$. Opt. Soc. Am. A 5, 30-38 (1988).

22. J.R. Fienup, "Reconstruction of an object from the modulus of its Fourier transform," Opt. Lett. 3, 27-29 (1978).

23. J. R. Fienup and C. C. Wackerman, "Phase retrieval stagnation problems and solutions," J. Opt. Soc. Am. A 3, 1897-1907 (1986).

24. J. N. Cederquist, J. R. Fienup, J. C. Marron, and R. G. Paxman, "Phase retrieval from experimental far-field speckle data," Opt. Lett. 13, 619-621 (1988).

25. R. H.T.Bates, W.R. Fright, and W.A. Norton, "Phase retrieval is successful in the optical as well as the computational laboratory," in Indirect Imaging, J. A. Roberts, ed. (Cambridge U. Press, Cambridge, 1983).

26. M. C. Won, D. Mnyama, and R. H. T. Bates, "Improving initial phase estimates for phase retrieval algorithms," Opt. Acta 32, 377-396 (1985).

27. P. S. Idell, J. D. Conglewski, and D. G. Voelz, "Image synthesis from nonimaged laser-speckle patterns: experimental verification," Opt. Lett. 14, 154-156 (1989).

28. P. S. Idell, J. R. Fienup, and S. Goodman, "Image synthesis from nonimaged laser-speckle patterns," Opt. Lett. 12, 858-860 (1987).

29. J. R. Fienup and P. S. Idell, "Imaging correlography with sparse array of detectors," Opt. Eng. 27, 778-784 (1988).

30. M. Nieto-Vesperinas and J. C. Dainty, "Phase recovery for twodimensional digital objects by polynomial factorization," Opt. Commun. 58, 83-88 (1986).

31. H. Szu and R. Hartley, "Fast simulated annealing," Phys. Lett. A 122, 157-162 (1987). 